\title{
ABSOLUTE AND RELATIVE PEAK POWER DURING PNEUMATIC SQUAT EXERCISE USING DIFFERENT PERCENTAGES OF LOAD IN ELITE SOCCER PLAYERS
}

original paper

( ) University School of Physical Education in Wroclaw

DOI: https://doi.org/10.5114/hm.2020.91347

\section{ALEX SOUTO MAIOR}

Augusto Motta University Centre, Rio de Janeiro, Brazil

\section{ABSTRACT}

Purpose. The study compared muscle peak power during pneumatic squat exercise with different percentages of load, corresponding to $80 \%, 100 \%$, and $120 \%$ of body mass (BM), in male professional soccer players.

Methods. The study involved 40 male elite soccer players $(25.2 \pm 3.6$ years; $179.6 \pm 6.1 \mathrm{~cm} ; 78.3 \pm 5.2 \mathrm{~kg}$; body fat $12.3 \pm$ $3.3 \%$ ) from a club of the Brazilian first division soccer league participating in national competitions organized by the Brazilian Soccer Confederation. Peak power was assessed by using a pneumatic squat machine (squat power test). The athletes applied different training loads during the squat power test ( 3 trials with $80 \%$ of BM followed by a 5 -minute rest to perform attempts with $100 \%$ and $120 \%$, respectively).

Results. The two-way ANOVA yielded main effects for group $\left(F_{3,26}=3.08, p<0.04\right)$ and for timing of measurement $F_{2,52}=526.1$, $p<0.0001$ ), indicating a significant difference in the absolute peak power of goalkeepers when compared with defenders and midfielders at the loads corresponding to $80 \%, 100 \%$, and $120 \%$ of BM. Intra-group comparison demonstrated a significant increase $(p<0.0001)$ in the absolute and relative peak power with loads corresponding to $120 \%$ of BM when compared with $80 \%$ and $100 \%$ of BM in all groups (defenders, goalkeepers, midfielders, and forwards).

Conclusions. The results confirm that loads corresponding to $120 \%$ of BM during pneumatic squat exercise contribute to greater absolute and relative peak power.

Key words: soccer players, peak power, squat exercise, performance

\section{Introduction}

Soccer is a sport of great popularity in the world, with 275 million participants of both sexes in a wide range of ages [1, 2]. A soccer match is characterized by jumping, confronting, running, passing, kicking the ball, with athletes required to perform quick, precise movements, rapid changes of direction, actions demanding deceleration and acceleration. Force development and muscle power are thus necessary to maintain optimal performance [3, 4].

It is evidenced that performance during explosive actions depends on muscle power, which may be determined by a number of neuromuscular variables, such as a combination of morphological and neural factors including muscle cross-sectional area and architecture, musculotendinous stiffness, motor unit re- cruitment, rate coding, motor unit synchronization, and neuromuscular inhibition [4-6]. Such combinations of factors are associated with enhanced external mechanical power, general sports skill performance, decreased injury rates, and training load monitoring [7].

An optimal training load during practice contributes to morphological, metabolic, and functional physiological adaptations that are advantageous when seeking successful competitive results [3, 8, 9]. This optimal training load is adjusted at various times during the training cycle to increase or maintain muscle power of athletes during the phase of training for playing soccer at a high level (i.e. baseline or competition phase). In addition, training load prescribing and monitoring are crucial for providing information on the efficacy of training doses and for supporting injury prevention strategies $[6,8,9]$. Thus, well-trained players gain an

Correspondence address: Alex Souto Maior, Augusto Motta University Centre - UNISUAM Postgraduate Program in Rehabilitation Sciences, Praça das Nações, 34 - Bonsucesso, ZIP Code 21041010 - Rio de Janeiro, RJ, Brazil, e-mail: alex.bioengenharia@gmail.com

Received: June 22, 2019

Accepted for publication: October 4, 2019

Citation: Maior Souto A. Absolute and relative peak power during pneumatic squat exercise using different percentages of load in elite soccer players. Hum Mov. 2020;21(3):64-70; doi: https://doi.org/10.5114/hm.2020.91347. 
edge in their performances as a result of periodized training procedures. But, what is the optimal training load for muscle power development in soccer players? Evidence of the training load monitoring in the buildup to a competitive athlete illustrates the importance of resistance training to the gain of muscle power used within soccer [10-13]. Nevertheless, the lack of studies which would characterize the optimal training load for muscle power development in soccer players still seems conflicting in the scientific literature. Some authors observed that a training load of $40-60 \%$ of 1-repetition maximum (1RM) during back squat exercise seemed to be effective to improve maximal power output in elite soccer players $[10,11]$. On the other hand, it was noted that strength training with a high intensity zone $(90 \%$ of $1 \mathrm{RM})$ was superior to a moderate intensity zone ( $70 \%$ of $1 \mathrm{RM})$ program because it increased strength without a change in muscle crosssectional area and improved repeated sprint ability [12]. However, a current study showed that 2 sessions per week of back squat training with loads of $70-90 \%$ of $1 \mathrm{RM}$ to the regular soccer training program of soccer players turned out sufficient to improve markers of soccer-related athletic performance such as sprint times, agility, jump, leg peak power, and the ability to make repeated changes of direction [13]. But the number of studies involving squat and pneumatic resistance in soccer players is limited.

Physical training with pneumatic resistance becomes effective by the relationship between force, acceleration, and mass in accordance with Newton's second law. Pneumatic equipment utilizes air pressure as a means of resistance, thereby reducing the mass component of the load to near zero. Thus, pneumatic resistance training may avoid the limitations of free weight by contributing to a load/resistance that is not subject to inertia $[4,6]$. But little is known about the use of $\%$ of body mass (BM) as an optimal training load during squat with pneumatic resistance equipment.

In this context, some authors have highlighted that the prescriptions of loads based on \% of BM can be efficient to increase muscle power in professional athletes [9]. Scientific literature shows that loads of $40-80 \%$ of BM during a jump squat exercise performed on a Smith machine may be efficient to increase muscle power in different sports [9]. Specifically, in elite soccer players, it was observed that no additional intensity zone and/or $40 \%$ of BM promoted improvements in speed during sprints of 5,10 , and $20 \mathrm{~m}$ and mean propulsive velocities during jump squat exercise [14]. However, few studies have examined the link between \% of BM, peak muscle power, and pneumatic squat exercise in elite soccer. Accordingly, the aim of this study was to compare the peak power muscle during pneumatic squat exercise with different percentages of load corresponding to \% of BM among male professional soccer players. It was hypothesized that loads $>100 \%$ of BM during pneumatic squat exercise would contribute to greater peak muscle power.

\section{Material and methods}

\section{Subjects}

The study included 40 male elite soccer players $(25.2 \pm 3.6$ years; $179.6 \pm 6.1 \mathrm{~cm} ; 78.3 \pm 5.2 \mathrm{~kg}$; body fat $12.3 \pm 3.3 \%$ ) from a club of the Brazilian first division soccer league participating in national competitions organized by the Brazilian Soccer Confederation. The players' training frequency was $6.3 \pm 1.2$ days/week, with training programs that consisted of plyometric training, ball possession, skipping, velocity, and resistance training. Exclusion criteria were as follows: (1) smoking history during the previous 3 months; (2) presence of a cardiovascular or metabolic disease; (3) systemic hypertension $(\geq 140 / 90 \mathrm{~mm} \mathrm{Hg}$ or use of antihypertensive medication); (4) use of anabolic steroids, drugs, or medication with the potential to impact physical performance (self-reported); (5) recent musculoskeletal injury; (6) symptoms of pain in any region of the body. All data collection was carried out at the beginning of training sessions during preseason.

Body composition was measured with bioelectrical impedance analysis (InBody720, Biospace, Seoul, Korea). The body composition analyser has in-built hand and foot electrodes. The subjects were dressed in underwear and barefooted, in the upright position, with their feet on the electrodes of the platform and their arms abducted with hands gripping the electrodes on the handles. All analyses were performed after 8 hours of fasting. All biometric measurements were carried out in an air-conditioned room $\left(21^{\circ} \mathrm{C}\right)$. No clinical problems occurred during the study.

\section{Squat power test}

Peak power was tested by using the Keiser Air 300 Squat Machine (Keiser, Fresno, USA), which is a pneumatic strength and power measurement machine. All athletes were tested in accordance with the specific position: defender $\times$ midfielder $\times$ forward $\times$ goalkeeper. The participants were already familiarized with the testing equipment because pneumatic resistance was 
part of the strength training program throughout the season. Prior to the squat power test, a 10-minute warm-up was performed that included 5 minutes of cycling at $50 \mathrm{~W}$ on a cycle ergometer, followed by 10 squats at $40 \%$ of the athletes' BM.

After the warm-up, the subjects were allowed 5 minutes of passive rest, followed by 3 maximal velocity trials during the squat power test, with a 1-minute rest between the trials. The athletes initiated the squat power test from a standing position, lowering to reach the starting position of $90^{\circ}$ of knee flexion, which was measured with a digital goniometer (Global Medical Devices; Maharashtra, India), with the load distributed across their shoulders. After maintaining the squat position for 3 seconds, the participants initiated the upper phase as quickly as possible back to the standing position without removing the heels from the floor.

The athletes were instructed to apply different training loads during the squat power test. All subjects started with $80 \%$ of BM; afterwards, the load was increased to $100 \%$ and $120 \%$ of BM. The rest between the sets with different training loads lasted for 5 minutes (i.e. 3 trials with $80 \%$ of $\mathrm{BM}$ followed by a 5 -minute rest to carry out the attempts with $100 \%$ and $120 \%$, respectively). The greatest peak power obtained in the 3 trials was used for the individual's comparison assessment.

\section{Statistical analysis}

Statistical analysis was initially performed with the Shapiro-Wilk normality tests and the homoscedasticity test (Bartlett criterion). Means and standard deviations were used to represent the centrality and spread of data. Comparisons between groups (defender $\times$ midfielder $\times$ forward $\times$ goalkeeper) and different training loads were performed by two-way ANOVA with Bonferroni post-hoc tests. In turn, intra-group comparison for each different training load was independently performed with one-way repeated-measures ANOVA followed by Tukey's post-hoc tests. The effect size was calculated to determine the significance of the difference by means of the $f 2$ for ANOVA. The level of significance for all statistical comparisons was set at $p<0.05$ by using $\mathrm{GraphPad}^{\circledR}$ software (Prism 6.0, San Diego, USA).

\section{Ethical approval}

The research related to human use has complied with all the relevant national regulations and institutional policies, has followed the tenets of the Declaration of Helsinki and the ethical standards in sport and exercise scienceresearch(CAE:76189817.0.0000. 5235), and has been approved by the local institutional Ethics Committee for Human Experiments.

\section{Informed consent}

Informed consent has been obtained from all individuals included in this study.

\section{Results}

All analysed data presented normal distribution. The two-way ANOVA yielded main effects for group $\left(F_{3,26}=3.08, p<0.04\right)$ and for timing of measurement $\left(F_{2,52}=526.1, p<0.0001\right)$; the Bonferroni posthoc $(p<0.05)$ showed significant differences in the absolute peak power of goalkeepers when compared with defenders and midfielders at the loads corresponding to $80 \%, 100 \%$, and $120 \%$ of the athletes' BM (Figure 1A). On the other hand, the two-way ANOVA revealed no significant differences in the relative peak power $\left(F_{6,52}=0.27, p<0.094\right)$ between the groups (Figure 1B).

(A)

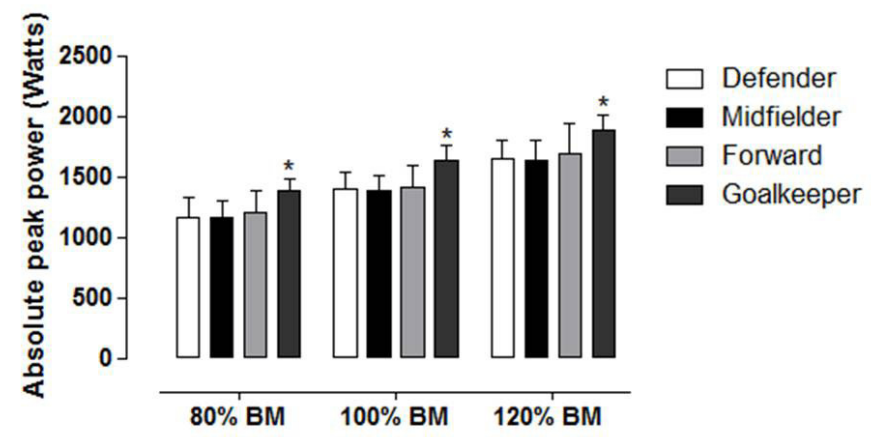

(B)

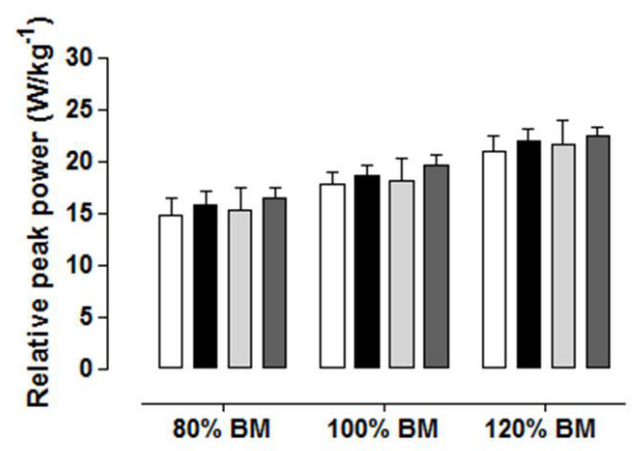

$\mathrm{BM}$ - body mass

${ }^{\star} p<0.05$ for goalkeeper $\times$ defender and midfielder groups

Figure 1. Mean \pm standard deviation values for comparisons between groups (defender $\times$ midfielder $\times$ forward $\times$ goalkeeper) and different training loads $(80 \% \times 100 \% \times 120 \%$ of body mass $)$ in professional soccer players 
(A)

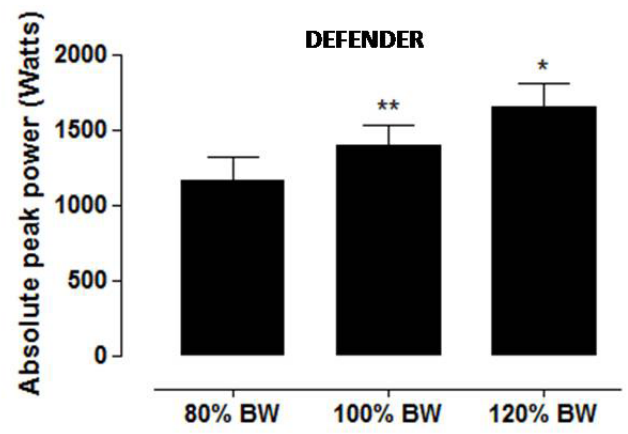

(C)

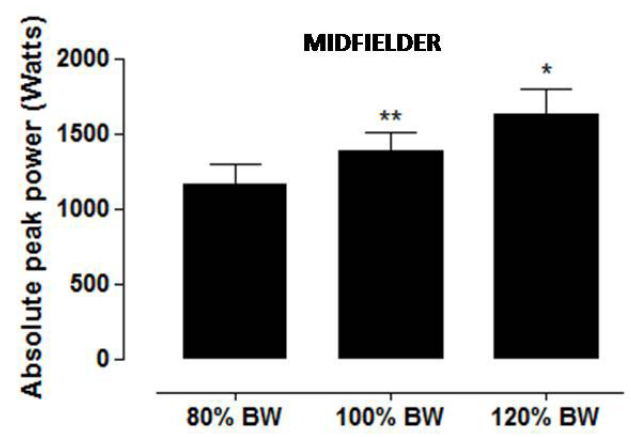

(B)

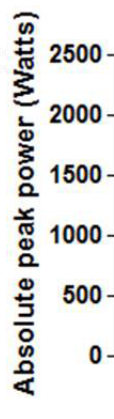

GOALKEEPER

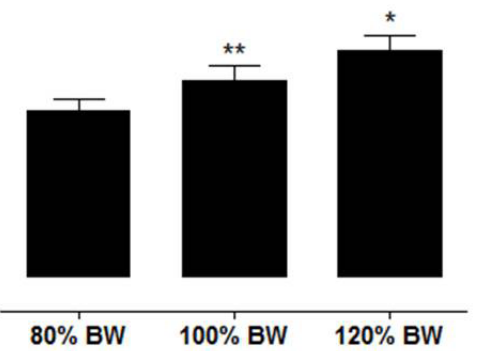

(D)

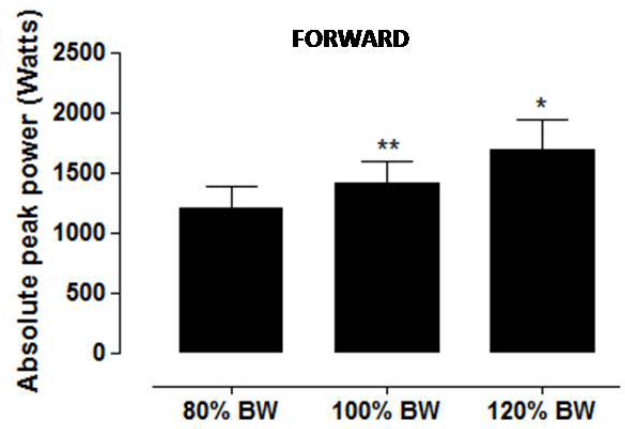

BW - body weight

${ }^{*} p<0.0001$ for $120 \% \times 80 \%$ and $100 \%$ of BW ${ }^{* *} p<0.0001$ for $100 \% \times 80 \%$ of BW

Figure 2. Mean \pm standard deviation values for intra-group comparisons of the absolute peak power with different training loads $(80 \% \times 100 \% \times 120 \%$ of body mass $)$ in professional soccer players

(A)

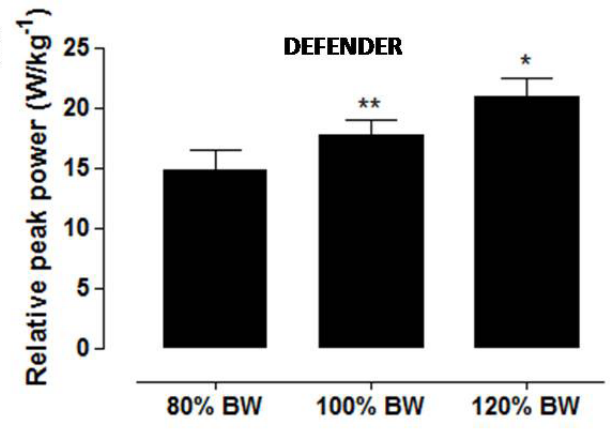

(C)

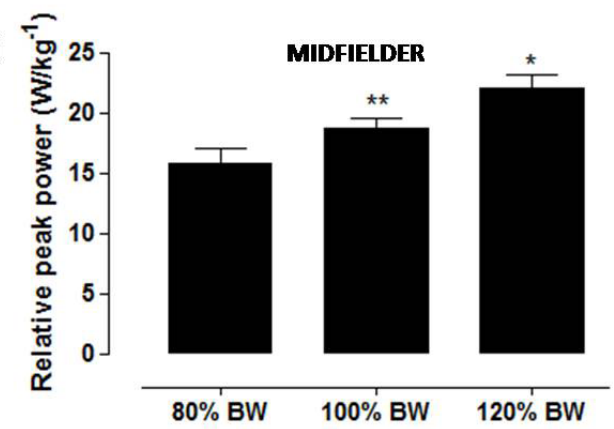

(B)

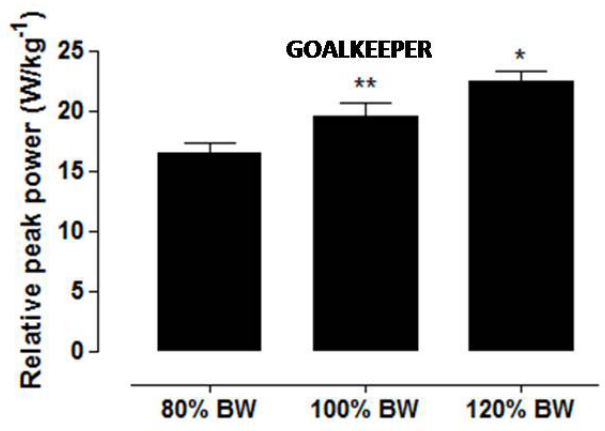

(D)

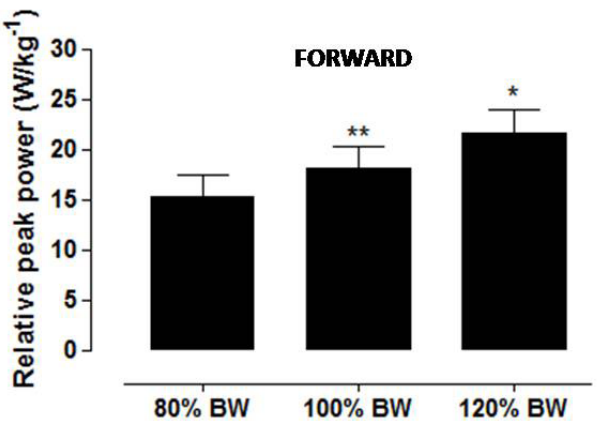

BW - body weight

${ }^{*} p<0.0001$ for $120 \% \times 80 \%$ and $100 \%$ of BW ${ }^{* *} p<0.0001$ for $100 \% \times 80 \%$ of BW

Figure 3. Mean \pm standard deviation values for intra-group comparisons of the relative peak power with different training loads $(80 \% \times 100 \% \times 120 \%$ of body mass $)$ in professional soccer players 
Absolute and relative peak power intra-group comparisons are presented in Figures 2 and 3. One-way ANOVA demonstrated a significant increase in the absolute and relative peak power at the loads corresponding to $120 \%$ of $\mathrm{BM}$ when compared with $80 \%$ and $100 \%$ of BM in defender (absolute: $F_{2,18}=16.86$, $p<0.0001, \eta^{2}=0.30$; relative: $F_{2,18}=8.74, p<0.0001$, $\eta^{2}=0.18$ ), goalkeeper (absolute: $F_{2,8}=47.78, p<0.0001$, $\eta^{2}=0.20$; relative: $F_{2,8}=38.76, p<0.0001, \eta^{2}=0.10$ ), midfielder (absolute: $F_{2,14}=20.13, p<0.0001, \eta^{2}=$ 0.29 ; relative: $F_{2,14}=9.10, p<0.0001, \eta^{2}=0.12$ ), and forward athletes (absolute: $F_{2,12}=26.07, p<0.0001$, $\eta^{2}=0.44$; relative: $F_{2,14}=27.67, p<0.0001, \eta^{2}=0.36$ ) (Figures 2 and 3). In addition, a significant increase in the absolute ( $p<0.0001$; Figure 2$)$ and relative $(p<0.0001$; Figure 3) peak power was evident at the loads corresponding to $100 \%$ when compared with $80 \%$ of $\mathrm{BM}$ in all groups of athletes.

\section{Discussion}

This study compared the peak muscle power during pneumatic squat exercise with different percentages of load, corresponding to \% of BM in male professional soccer players. Our findings support the original hypothesis that loads $>100 \%$ of $\mathrm{BM}$ during pneumatic squat exercise contribute to greater absolute and relative peak muscle power. Indeed, the notable results evidenced in the present study show that loads corresponding to $120 \%$ of $\mathrm{BM}$ resulted in an increase of absolute and relative peak muscle power in all groups of professional soccer players. But, absolute peak muscle power of goalkeepers was greater when compared with the defender and midfielder groups. No studies were found that would evaluate the peak muscle power during pneumatic squat exercise with different percentages of load corresponding to \% of BM among male professional soccer players.

Pneumatic resistance is not related to the inertia and momentum of the load, thus limiting the effect of velocity variations, becoming constant throughout the movement and leading to greater mean power $[15,16]$. Indeed, this exhibits less variation in the muscular force required to complete a resistance training program and, consequently, reduces the risk of muscle injury [17]. Evaluation of peak power using pneumatic equipment has recently been applied as a reliable muscle power assessment method because this equipment can accurately measure the force and velocity components of muscle power output [15, 16]. Peak power is an instantaneous measure of the optimal forcevelocity combination at a specific point during the movement. Several studies evaluated peak power muscle with \% of 1RM [10, 12, 13]; however, no research utilized loads corresponding to \% of BM.

A study mentioned that during squat exercise, for increasing peak power, a weightlifter must train with a load of $80-100 \%$ of $1 \mathrm{RM}$, whereas a sprinter or jumper can increase performance with a training load of $10-50 \%$ of $1 \mathrm{RM}$ [18]. But, the greatest peak power values were associated with loads of $40 \%$ and $50 \%$ of 1RM during standard parallel squat [19]. In particular, professional soccer players revealed improvement in the power output during preseason with an optimal resistance training load of $45 \%$ of $1 \mathrm{RM}(30-60 \%$ of 1RM) [11]. Conversely, soccer players who trained twice a week during 10 weeks performing half-squat with a fixed external load of $65 \%$ of $1 \mathrm{RM}$ showed an increase in absolute $( \pm 11 \%)$ and relative $( \pm 11.2 \%)$ peak power [20]. Contrary to our results, another study reported that a load corresponding to $112.5 \%$ of BM $(60.3 \%$ of $1 \mathrm{RM})$ contributed to maximum peak power $(1148.6 \pm$ $301 \mathrm{~W}$ ) in professional soccer players; however, halfsquat tests were performed on a Smith machine [21]. Our results verified that a load corresponding to $120 \%$ of BM reached $1694 \pm 192 \mathrm{~W}$ of maximal peak power during squat with pneumatic resistance equipment, while another study [21] showed that a load of $125 \%$ of BM attained values of $1033.5 \mathrm{~W}$ with a Smith machine. The difference of $\pm 39 \%$ in the maximal peak power between squat exercises with the pneumatic resistance equipment vs. Smith machine may be related to improvements in peak power and power endurance owing to the requirement of pneumatic resistance equipment in eccentric muscle actions of the exercise and an increase of the hip and thigh power production during the concentric phase [15, 16]. A greater eccentric muscle action in leg muscle contributes to a better transition of the extensor muscles to explosive power $[22,23]$. The performance improvement may occur by lower limb resistance training and a control of the training load [24]. Thus, coaches must understand the need of appropriate training load monitoring to increase relative and absolute peak power output.

\section{Limitations}

The limitations of the study include (1) the absence of measures of physiological parameters of physical exertion, which would be interesting; this, yet, does not limit the answer to the study question; and (2) lack of assessments of the angulations in knee flexion during pneumatic squat exercise. Additionally, it is suggested that future studies evaluate the physiological 
effect, angulations of different knee flexions, and perception of effort.

\section{Conclusions}

The results of this investigation confirm that loads $>100 \%$ of BM during pneumatic squat exercise contribute to greater absolute and relative peak muscle power. In particular, professional soccer players showed improvements in the peak power with loads corresponding to $120 \%$ of BM. It is recommended to use this optimal training load as it might be counterproductive to the muscle power gains.

\section{Acknowledgments}

The investigator would like to thank the 40 healthy male professional soccer players from a club in the Brazilian first division that participates in national and international competitions organized by the Brazilian Soccer Confederation and South American Soccer Confederation. The study was supported by the Coordenação de Aperfeiçoamento de Pessoal de Nível Superior, Brazil (CAPES), Finance Code 001.

\section{Disclosure statement}

The author does not have any financial interest and did not receive any financial benefit from this research.

\section{Conflict of interest}

The author states no conflict of interest.

\section{References}

1. Ekstrand J, Hägglund M, Waldén M. Injury incidence and injury patterns in professional football: the UEFA injury study. Br J Sports Med. 2011;45(7):553-558; doi: 10.1136/bjsm.2009.060582.

2. Pfirrmann D, Herbst M, Ingelfinger P, Simon P, Tug S. Analysis of injury incidences in male professional adult and elite youth soccer players: a systematic review. J Athl Train. 2016;51(5):410-424; doi: 10.4085/10626050-51.6.03.

3. Souto Maior A, Viana J, Hall E, Ferreira AS, Bezerra E. Correlation between match efforts and serum creatine kinase level: implications for injury prevention in elite soccer players. JEPonline. 2018;21(5):109-120.

4. Cormie P, McGuigan MR, Newton RU. Developing maximal neuromuscular power: Part 1- biological basis of maximal power production. Sports Med. 2011; 41(1):17-38; doi: 10.2165/11537690-000000000-00000.

5. Methenitis S, Karandreas N, Spengos K, Zaras N, Stasinaki AN, Terzis G. Muscle fiber conduction velocity, muscle fiber composition, and power performance. Med Sci Sports Exerc. 2016;48(9):1761-1771; doi: 10.1249/ MSS.0000000000000954.
6. Suchomel TJ, Nimphius S, Bellon CR, Stone MH. The importance of muscular strength: training considerations. Sports Med. 2018;48(4):765-785; doi: 10.1007/ s40279-018-0862-z.

7. Suchomel TJ, Nimphius S, Stone MH. The importance of muscular strength in athletic performance. Sports Med. 2016;46(10):1419-1449; doi: 10.1007/ s40279-016-0486-0.

8. Casamichana D, Castellano J, Calleja-Gonzalez J, San Román J, Castagna C. Relationship between indicators of training load in soccer players. J Strength Cond Res. 2013;27(2):369-374; doi: 10.1519/JSC.0b013e318254 8 af1.

9. Loturco I, Pereira LA, Kobal R, Kitamura K, Cal Abad CC, Nakamura FY, et al. Peak versus mean propulsive power outputs: which is more closely related to jump squat performance? J Sports Med Phys Fitness. 2017; 57(11):1432-1444; doi: 10.23736/S0022-4707.17.069 40-7.

10. De Hoyo M, Gonzalo-Skok O, Sañudo B, Carrascal C, Plaza-Armas JR, Camacho-Candil F, et al. Comparative effects of in-season full-back squat, resisted sprint training, and plyometric training on explosive performance in U-19 elite soccer players. J Strength Cond Res. 2016;30(2):368-377; doi: 10.1519/JSC.0000000 000001094.

11. Loturco I, Ugrinowitsch C, Tricoli V, Pivetti B, Roschel H. Different loading schemes in power training during the preseason promote similar performance improvements in Brazilian elite soccer players. J Strength Cond Res. 2013;27(7):1791-1797; doi: 10.1519/JSC. 0b013e3182772da6.

12. Bogdanis GC, Papaspyrou A, Souglis AG, Theos A, Sotiropoulos A, Maridaki M. Effects of two different half-squat training programs on fatigue during repeated cycling sprints in soccer players. J Strength Cond Res. 2011;25(7):1849-1856; doi: 10.1519/JSC.0b013e3181 e83a1e.

13. Hammami M, Negra Y, Billaut F, Hermassi S, Shephard RJ, Chelly MS. Effects of lower limb strength training on agility, repeated sprinting with changes of direction, leg peak power, and neuromuscular adaptations of soccer players. J Strength Cond Res. 2018;32(1):3747; doi: 10.1519/JSC.0000000000001813.

14. Loturco I, Nakamura FY, Kobal R, Gil S, Cal Abad CC, Cuniyochi R, et al. Training for power and speed: effects of increasing or decreasing jump squat velocity in elite young soccer players. J Strength Cond Res. 2015; 29(10):2771-2779; doi:10.1519/JSC.0000000000000 951.

15. Frost DM, Cronin JB, Newton RU. A comparison of the kinematics, kinetics and muscle activity between pneumatic and free weight resistance. Eur J Appl Physiol. 2008;104(6):937-956; doi: 10.1007/s00421008-0821-8.

16. Frost DM, Bronson S, Cronin JB, Newton RU. Changes in maximal strength, velocity, and power after 8 weeks 


\section{HUMAN MOVEMENT}

A. Souto Maior, Peak power in elite soccer players

of training with pneumatic or free weight resistance. J Strength Cond Res. 2016;30(4):934-944; doi: 10.1519/ JSC.0000000000001179.

17. Frost DM, Cronin J, Newton RU. A biomechanical evaluation of resistance: fundamental concepts for training and sports performance. Sports Med. 2010;40(4):303326; doi: 10.2165/11319420-000000000-00000.

18. McBride JM, Haines TL, Kirby TJ. Effect of loading on peak power of the bar, body, and system during power cleans, squats, and jump squats. J Sports Sci. 2011; 29(11):1215-1221; doi: 10.1080/02640414.2011.587444.

19. Zink AJ, Perry AC, Robertson BL, Roach KE, Signorile JF. Peak power, ground reaction forces, and velocity during the squat exercise performed at different loads. J Strength Cond Res. 2006;20(3):658-664; doi: 10.1519/ R-16264.1.

20. Ramírez JM, Núñez VM, Lancho C, Poblador MS, Lancho JL. Velocity-based training of lower limb to improve absolute and relative power outputs in concentric phase of half-squat in soccer players. J Strength Cond Res. 2015;29(11):3084-3088; doi: 10.1519/JSC.0000 000000000407.

21. Requena B, González-Badillo JJ, de Villareal ES, Ereline J, García I, Gapeyeva H, et al. Functional performance, maximal strength, and power characteristics in isometric and dynamic actions of lower extremities in soccer players. J Strength Cond Res. 2009;23(5): 1391-1401; doi: 10.1519/JSC.0b013e3181a4e88e.

22. Stasinaki AN, Zaras N, Methenitis S, Bogdanis G, Terzis G. Rate of force development and muscle architecture after fast and slow velocity eccentric training. Sports. 2019;7(2):41; doi: 10.3390/sports7020041.

23. Kabaciński J, Murawa M, Fryzowicz A, Dworak LB. A comparison of isokinetic knee strength and power output ratios between female basketball and volleyball players. Hum Mov. 2017;18(3):40-45; doi: 10.1515/ humo-2017-0022.

24. Clemente FM, Nikolaidis PT, Van Der Linden CMIN, Silva B. Effects of small-sided soccer games on internal and external load and lower limb power: a pilot study in collegiate players. Hum Mov. 2017;18(1):50-57; doi: 10.1515/humo-2017-0007. 